\title{
Production of Bio-Based Isoprene by the Mevalonate Pathway Cassette in Ralstonia eutropha ${ }^{\text {S }}$
}

\author{
Hyeok-Won Lee ${ }^{1 \dagger}$, Jung-Ho Park ${ }^{2 \dagger}$, Hee-Seok Lee ${ }^{1,3}$, Wonho Choi ${ }^{2}$, Sung-Hwa Seo ${ }^{1}$, Irika Devi Anggraini ${ }^{1,3}$, \\ Eui-Sung $\mathrm{Choi}^{1,3}$, and Hong-Weon Lee ${ }^{1,3 *}$ \\ ${ }^{1}$ Biotechnology Process Engineering Center, Korea Research Institute of Bioscience and Biotechnology (KRIBB), Cheongju 28116, Republic of Korea \\ ${ }^{2}$ Bio-Evaluation Center, Korea Research Institute of Bioscience and Biotechnology (KRIBB), Cheongju 28116, Republic of Korea \\ ${ }^{3}$ Department of Bioprocess Engineering, University of Science and Technology (UST) of Korea, Daejeon 34113, Republic of Korea
}

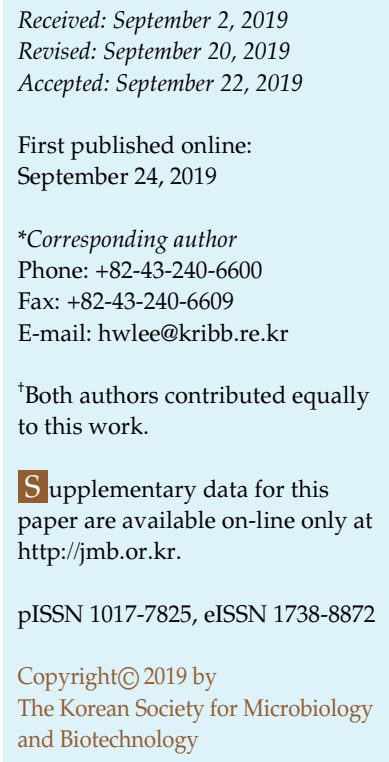

Isoprene has the potential to replace some petroleum-based chemicals and can be produced through biological systems using renewable carbon sources. Ralstonia eutropha can produce value-added compounds, including intracellular polyhydroxyalkanoate (PHA) through fatty acid and lipid metabolism. In the present study, we engineered strains of R. eutropha H16 and examined the strains for isoprene production. We optimized codons of all the genes involved in isoprene synthesis by the mevalonate pathway and manipulated the promoter regions using pLac and pJ5 elements. Our results showed that isoprene productivity was higher using the J5 promoter $(1.9 \pm 0.24 \mu \mathrm{g} / \mathrm{l})$ than when using the lac promoter $(1.5 \pm 0.2 \mu \mathrm{g} / \mathrm{l})$. Additionally, the use of three J5 promoters was more efficient $(3.8 \pm 0.18 \mu \mathrm{g} / 1)$ for isoprene production than a one-promoter system, and could be scaled up to a 5-L batch-cultivation from a T-flask culture. Although the isoprene yield obtained in our study was insufficient to meet industrial demands, our study, for the first time, shows that $R$. eutropha can be modified for efficient isoprene production and lays the foundation for further optimization of the fermentation process.

Keywords: Ralstonia eutropha, isoprene production, promoter, fermentation, MVA pathway

\section{Introduction}

Ralstonia eutropha is an industrial strain used in the production of various industrial materials, biodegradable plastics, biofuels as alternatives to gasoline, and other biobased chemicals because of the ability of $R$. eutropha to use a sole carbon source such as sugar, amino acid, carbon dioxide, fatty-acids, and various oils for cell growth [1-4]. Specifically, R. eutropha is known as a model bacterium for biodegradable polyhydroxyalkanoate (PHA) biopolymer production [5-7]. However, $\mathrm{PHB}$, a type of $\mathrm{PHA}$, has been shown to accumulate up to $70 \%$ of dry cell weight (DCW) by adequate carbon metabolism under unfavorable culture conditions of nutrient limitation [8]. Recently, R. eutropha has gained much attention as a candidate for biofuel production, which is an alternative to petroleum-based products. The bacterium can be engineered to produce, $\mathrm{C} 4$ and C5 alcohols such as isobutanol, and 3-methyl-1butanol by electromicrobial conversion of $\mathrm{CO}_{2}$ [9]. In addition, R. eutropha has been used to produce various industrial materials including isopropanol, methyl ketone, and isoprene using acetyl-CoA as a precursor of PHA biosynthesis. Batch cultivation of R. eutropha (Re2133/ pEG7c) has been carried out using fructose as a sole carbon source to produce $3.44 \mathrm{~g} / 1$ isopropanol [10]. In the case of medium-chain hydrocarbons, $180 \mathrm{mg} / 1$ methyl ketones were obtained by heterologous expression of the acyl coenzyme A oxidase under chemolithoautotrophic growth conditions with $\mathrm{CO}_{2}$ and $\mathrm{H}_{2}$ as the sole carbon source and electron donor, respectively [11].

Isoprene hydrocarbons are a useful chemical product widely used as feedstock in fuel additives for gasoline or jet fuel as well as in the synthetic rubber industry [12-14]. The production of isoprenoids including isoprene is 
divided into two biosynthetic pathways, the mevalonate (MVA) pathway and the methylerythritol phosphate (MEP) pathway from isopentenyl diphosphate pyrophosphate (IPP) and dimethylallyl diphosphate (DMAPP), with each having been studied using engineered E. coli systems [1517]. Several reports have also described bio-based isoprene production using novel engineered strains of Bacillus, cyanobacteria, and Saccharomyces cerevisiae species [18, 19]. The MVA pathway is used by eukaryotes and archaebacteria, and occurs in the cytosols of higher plants, while the MEP pathway is mainly used by eubacteria and green algae [20, 21]. The MVA pathway requires acetyl-CoA as the primary precursor, whereas the MEP pathway requires pyruvate and glyceraldehyde-3-phosphate as initial molecules for IPP and DMAPP biosynthesis. The MEP pathway has been the target of genetic engineering in efforts to increase the supply of IPP and DMAPP substrates for increased synthesis of isoprenoids [22, 23]. However, this approach still has limitations because the MEP pathway may be associated with unknown physiological regulation elements in the native host [24]. Heterologous expression of the MVA pathway can contribute to the increase in metabolic flux and synthesis of IPP and DMAPP and can be used as an alternative to the MEP pathway [25].

In our previous study, we achieved $12.7 \mathrm{~g} / 1$ isoprene accumulation through heterologous co-expression of the Populus trichocapa ispS gene and MVA pathway genes using E. coli DH5 $\alpha$ [17]. Despite the use of various hosts, genetic elements, and culture conditions in previous studies, the yield produced is still insufficient to meet the industrial demand for cost-effective productivity.

Although R. eutropha appears to be a suitable host for isoprene production due to its autotrophic growth, it has never been engineered as a host strain for this purpose. Therefore, we demonstrate here for the first time the production of isoprene using the MVP pathway in R. eutropha H16. A three-promoter system was introduced for isoprene production by the mevalonate pathway. In previous reports, the expression level of eGFP under various promoters has been evaluated in R. eutropha H16 transconjugants, and the results showed that eGFP expression using the $\mathrm{J} 5$ promoter was 5 times higher than that using the lac promoter [26]. Further, highly efficient overproduction of the E. coli protein complex has been achieved using a two-promoter system [27]. To improve isoprene production, all the genes involved in isoprene synthesis by the mevalonate pathway were optimized according to the codon usage of R. europha, and a novel cassette with Plac and PJ5 promoter elements was used.

\section{Materials and Methods}

\section{Bacterial Strains and Plasmids}

Bacterial strains and plasmids used in this study are listed in Table 1. E. coli S17 and R. eutropha H16 harboring pTS-sPt-MVA were used in this study. The plasmid was obtained from pS-NA plasmid derived from pSTV28, as described previously [17] and contained the following: mvaS and hydroxymethylglutaryl-CoA reductase (mvaE) from Enterococcus faecalis; mevalonate kinase (mvaK1), phosphomevalonate kinase (mvaK2), and mevalonate diphosphate decarboxylase (mvaD) from Streptococcus pneumoniae; idi from E. coli; and ispS from Populus trichocarpa. ispS is an isoprene synthase gene to catalyze the formation of isoprene from DMAPP. All plasmids constructed in this study contain the backbone from the broad-host-range vector pBBR1-MCS2 with the kanamycin resistance gene [28]. pBBR1MCS-2- $\mathrm{P}_{\mathrm{J5}}$ was constructed by replacing the $\mathrm{P}_{\mathrm{LAC}}$ promoter region of the pBBR1MCS-2 vector, and the sequences of the promoters and terminators are listed in Table S1. DNA sequence amplification was achieved using Phusion Flash High-Fidelity PCR Master Mix (New England Biolabs, USA). Restriction enzymes were obtained from New England Biolabs. The primers used in this study are listed in Table S2, and all constructed plasmids are described in Table 1. Codon usage tables of mevalonate pathway and isoprene synthase genes in $R$. eutropha are listed in Table S3.

\section{Culture Conditions}

Conjugated R. eutropha strains for isoprene production were pre-cultured in $2 \mathrm{ml}$ of tryptic soy broth (TSB) supplemented with $10 \mu \mathrm{g} / \mathrm{ml}$ gentamicin for $24 \mathrm{~h}$ at $30^{\circ} \mathrm{C}$. The pre-cultured cells were transferred to a $250-\mathrm{ml}$, sealed T-flask containing $20 \mathrm{ml}$ of TSB broth with $2 \mathrm{~g} / 1$ fructose to compare isoprene production of conjugated R. eutropha cells. For 5-L batch fermentation, the seed media were transferred to a 5 - $\mathrm{L}$ fermenter containing $100 \mathrm{ml}$ of TSB broth as a seed culture. After $12 \mathrm{~h}$ of cultivation at $37^{\circ} \mathrm{C}$ on a rotary shaker $(200 \mathrm{rpm})$, the culture was inoculated into a 5-L fermenter (KFC LA-150; Kobiotech Co., Ltd., Korea) containing $2 \mathrm{~L}$ of the initial medium [ $20 \mathrm{~g} / 1$ fructose, $20 \mathrm{~g} / 1$ yeast extract, $10 \mathrm{~g} / 1$ tryptone peptone, $5 \mathrm{~g} / 1\left(\mathrm{NH}_{4}\right)_{2} \mathrm{SO}_{4}, 3 \mathrm{~g} / 1 \mathrm{KH}_{2} \mathrm{PO}_{4}, 3 \mathrm{~g} / 1 \mathrm{Na}_{2} \mathrm{HPO}_{4}$, $1 \mathrm{~g} / 1 \mathrm{MgSO}_{4} \cdot 7 \mathrm{H}_{2} \mathrm{O}, 0.4 \mathrm{ml} / \mathrm{l}$ antifoam, $10 \mathrm{mg} / \mathrm{ml}$ gentamicin] and $1 \mathrm{ml}$ of trace element solution. The trace element solution was prepared as previously described [29]. The phosphate-containing compounds and magnesium sulfate were sterilized separately from the main medium. Aeration was maintained at $0.2 \mathrm{vvm}$ and the stirring speed was gradually increased to $200-1,000 \mathrm{rpm}$ to maintain the dissolved oxygen (DO) concentration at $\geq 20 \%$ by adjusting the rate of agitation and maintaining the $\mathrm{pH}$ at 7.0 with $10 \mathrm{~N} \mathrm{NaOH}$. For 5-L batch cultivation using the three-promoter system, cell growth was monitored by measuring the optical density at $600 \mathrm{~nm}$, starting from an initial value of 0.42 . 
Table 1. Bacterial strains and plasmids used in this study.

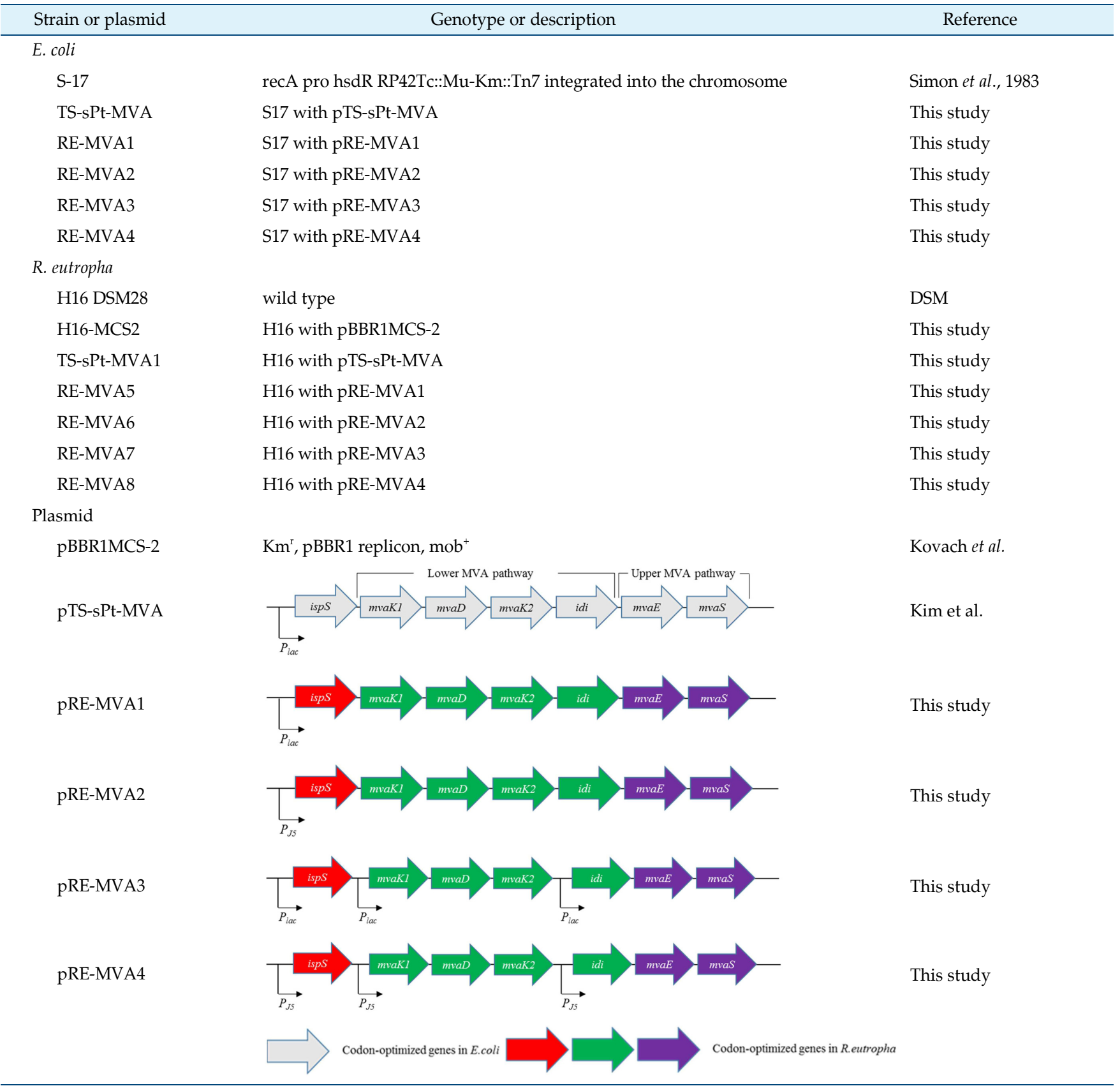

\section{Analytical Methods}

An auto-sampler (CNS, Korea) was used to measure both the optical density and the fructose concentration in the culture broth at specified time points. Cell growth was monitored by measuring the $\mathrm{OD}$ at $600 \mathrm{~nm}\left(\mathrm{OD}_{600}\right)$ using a spectrophotometer (Uvicon 941 Plus; Kontron Instruments Co., Switzerland). The residual fructose concentration was analyzed on a high-performance liquid chromatography (HPLC) system with an RID detector (RID-7515A, ERC Instrument Co., Japan) equipped with an
Aminex 87H ion-exclusion column (Bio-Rad, Hercules, CA, USA). The column temperature was maintained at $85^{\circ} \mathrm{C}$, and the mobile phase was deionized water applied at a flow rate of $0.5 \mathrm{ml} / \mathrm{min}$. The isoprene concentration was measured with a gas chromatograph (Varian X-3300, USA) equipped with a flame ionization detector. The analysis was performed at previously reported conditions [17]. The temperature program used was $3 \mathrm{~min}$ at $50^{\circ} \mathrm{C}$ followed by an increase to $150^{\circ} \mathrm{C}$ for $10 \mathrm{~min}$; the column was maintained at this temperature for $12 \mathrm{~min}$ before lowering to $50^{\circ} \mathrm{C}$ again. Isoprene 
production was identified using a gas chromatography-mass spectrometry (GC-MS) system (Agilent GC/MSD 5975 coupled with an Agilent 7890A) equipped with a quadrupole mass selective detector on electro-ionization (EI) operated at $70 \mathrm{eV}$. The sim-mode was used to detect the desired peaks. The Agilent HP5MS column ( $30 \mathrm{~m}$ length, $0.25 \mu \mathrm{m}$ inner diameter, $0.25 \mu \mathrm{m}$ film thickness) was used at a 10:1 split ratio, helium was used as a carrier gas, and the oven temperature ranged from 150 to $172^{\circ} \mathrm{C}$.

\section{Nucleotide Accession Numbers}

The ispS, mvaK1, mvaD, mvaK2, idi, mvaE, mvaS, and LipA DNA sequences codon-optimized in R. eutropha have been deposited at GenBank under the accession numbers of MK775336, MK775337, MK775338, MK775339, MK775340, MK775341, MK775342, and MK775343, respectively.

\section{Results}

Identification of Isoprene Production Using GC and GCMS from T-Flask Cultivation of Engineered R. eutropha Strains

Vapor samples from the sealed T-flask cultivation of engineered $R$. eutropha strains carrying the plasmid (pREMVA5 to -MVA8) with the $\mathrm{P}_{\mathrm{L}}$ (lac promoter), $\mathrm{P}_{\mathrm{J}}$ (J5 promoter), $\mathrm{P}_{\mathrm{LLL}}$ (three lac promoters), and $\mathrm{P}_{\mathrm{JJJ}}$ (three J5 promoters) were examined by GC and GC-MS techniques (Table 1). For GC-MS analysis, $1 \mathrm{ml}$ of gas from the headspace of sealed cultures was manually injected into the GC inlet port, based on a calibration curve constructed from serial dilutions of vaporized isoprene standard. For the calibration
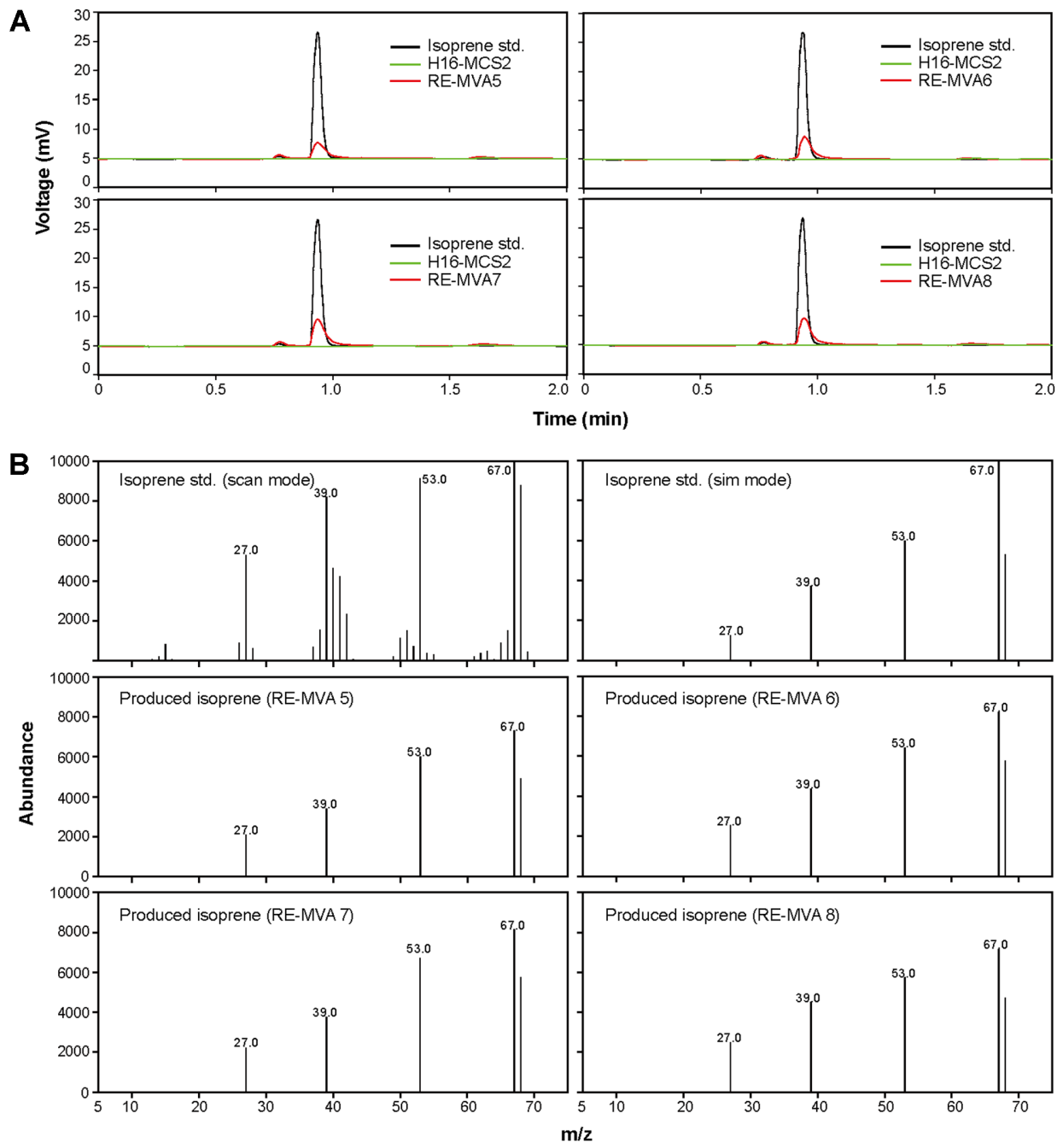

Fig. 1. Production of isoprene in R. eutropha.

Detection of produced isoprene by (A) GC peak, (B) GC-Mass peak during T-flask cultivation of recombinant $R$. eutropha strains harboring plasmids pTS-sPT-MVA, pRE-MVA1, pRE-MVA2, pRE-MVA3, and pRE-MVA4. 
curve, a $1 \mathrm{ml}$ sample from diluted standard was injected in the GC and the peak amplitude of the sample was recorded (Fig. 1A). The GC peak areas were plotted against the known amount of isoprene, and the slope of the standard curve was used to determine the amount of isoprene produced by the engineered $R$. eutropha strains in T-flask and 5 -L batch cultivation. The headspace samples including isoprene standard showed two peaks with retention times of 0.75 and $0.94 \mathrm{~min}$ (Fig. 1A). For more accurate identification of the isoprene produced by the recombinant R. eutropha strains, the samples were subjected to GS-MS. The Agilent GC/MSD 5975 was coupled with an Agilent 7890A device, which allowed selection of scanning or selected ion monitoring (SIM) mode. The scan mode photograph showed all ionized isoprene peaks having a molecular weight of 68.1 in reference (Fig. 1B). The scan mode can represent a mixed spectrum of the target and complex compounds. On the other hand, the chromatographic run of SIM-scan mode has the ability to obtain powerful information at very low detection limits of the target compound. Therefore, the SIM mode of GC-MS was used to identify the isoprene produced by the recombinant R. eutropha strains. GC-MS analysis of vapor samples from the headspace of the transformants is shown in Fig. 1B. The peaks displayed dominant 27.0, 39.0, 53.0, and 67.0 lines, which could be attributed to isoprene. The isoprene from all four $R$. eutropha transformants examined appeared as separate and distinct GC-MS peaks (Fig. 1B). Together, these results clearly showed that the recombinant $R$. eutropha could actively produce isoprene as well as various biobased products.

\section{Cultivation of R. eutropha H16 Transconjugants Harboring Each of Five Plasmids}

In order to achieve optimal carbon flow into isoprene biosynthetic pathway, R. eutropha H16 strains harboring mevalonate pathway genes and isoprene synthase were constructed. The strains H16-MCS2 and TS-sPt-MVA1 were conjugated with the plasmids codon-optimized in E. coli, namely, pBBR1MCS2 and pTS-sPt-MVA, respectively, as control strains for isoprene production in this study. All strains were cultivated on fructose $(2 \mathrm{~g} / \mathrm{l}$, for protection against decrease in $\mathrm{pH}$ ) as the sole carbon source with TSB media without dextrose during the sealed T-flask cultures. As shown in Fig. 2, isoprene was not detectable in the H16MCS2 strain transformed with the empty plasmid pBBR1MCS2. In the case of TS-sPt-MVA1 strain harboring pTS-sPt-MVA, the isoprene production $(0.45 \pm 0.16 \mu \mathrm{g} / \mathrm{l})$ was slightly lower than that in the strains harboring codon- optimized genes in R. eutropha in the T-flask cultivation. Next, we compared the efficiency of the Lac and J5 promoters by comparing the expression level of alkaline lipase (LipA). LipA derived from Proteus vulgaris consists of 287 amino acids. R. eutropha H16 transformants containing pLac-LipA and pJ5-LipA were screened using 1\% tributyrin-emulsion YPD plates using fructose instead of glucose [30]. The clear zone on the opaque tributyrin emulsion identified the lipase-secreting transformants. A clear zone was formed around pLac-LipA and pJ5-LipA fermentation broth and supernatant after cell disruption. The diameter of the transparent ring formed by the $\mathrm{pJ} 5$-LipA was larger than that formed by the pLac-LipA, indicating that the J5 promoter was the stronger of the two promoters in $R$. eutropha (Fig. S1). The isoprene produced by the R. eutropha conjugates with the one-promoter system of pLac and pJ5 was $1.5 \pm 0.2 \mu \mathrm{g} / 1$ and $1.9 \pm 0.24 \mu \mathrm{g} / \mathrm{l}$, respectively (Fig. 2). We also examined the efficacy of a three-promoter system of $\mathrm{P}_{\mathrm{LLL}}$ and $\mathrm{P}_{J J J}$ for isoprene production (Table 1). The $\mathrm{P}_{\mathrm{JJJ}}$ system showed higher isoprene production than $\mathrm{P}_{\mathrm{LLL}}$ in sealed T-flask cultivation, and the amounts of isoprene produced by $\mathrm{P}_{\mathrm{JJJ}}$ and $\mathrm{P}_{\mathrm{LLL}}$ were $3.4 \pm 0.05 \mu \mathrm{g} / 1$ and $3.8 \pm 0.18 \mu \mathrm{g} / \mathrm{l}$, respectively. In addition, the specific yield and carbon yield of the three-promoter system was higher than that of the one-promoter system. The maximum specific yield (2.53 $\mu \mathrm{g}$ isoprene/OD) and carbon yield (1.9 $\mu \mathrm{g}$ isoprene/g c-source) was obtained by the strain containing the $\mathrm{P}_{\text {JJJ }}$ promoter (Fig. 2). The results of the sealed T-flask cultivation are summarized in Table 2.

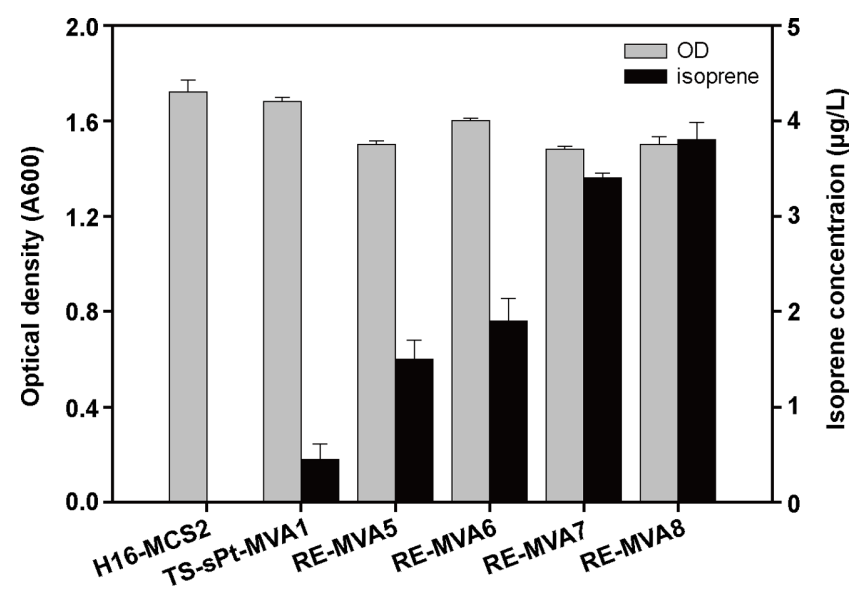

Fig. 2. T-flask cultivation of R. eutropha H16.

R. eutropha $\mathrm{H} 16$ transconjugants harboring plasmids pTS-sPT-MVA, pRE-MVA1, pRE-MVA2, pRE-MVA3, and pRE-MVA4. Error bars indicate $\mathrm{SD} ; n=3$ experiments. 
Cultivation of R. eutropha H16 Transconjugants Containing Three-Promoter Systems in 5-L Batch Cultivation

As described above, R. eutropha H16 harboring the threepromoter system demonstrated the highest isoprene productivity in sealed T-flask cultures. Therefore, these strains were further evaluated as candidate strains for isoprene production by 5 - $\mathrm{L}$ batch culture. Fructose and $\mathrm{NaOH}(10 \mathrm{~N})$ were used as the single carbon source and the $\mathrm{pH}$ control reagent, respectively. The time profiles for isoprene production by $R$. eutropha $\mathrm{H} 16$ was determined in the complex culture medium (Fig. 3). To improve the isoprene detection using on-line GC, $0.2 \mathrm{vvm}$ of aeration and the DO level $(20 \%)$ were maintained with agitation. The lag phase was terminated after approximately $6 \mathrm{~h}$ of culture, and cell growth entered the exponential growth

A

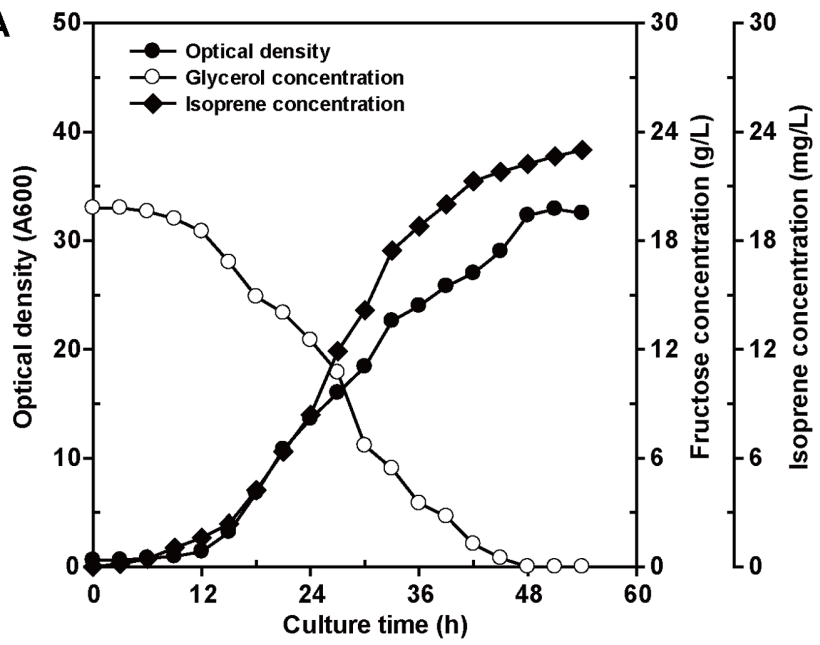

B

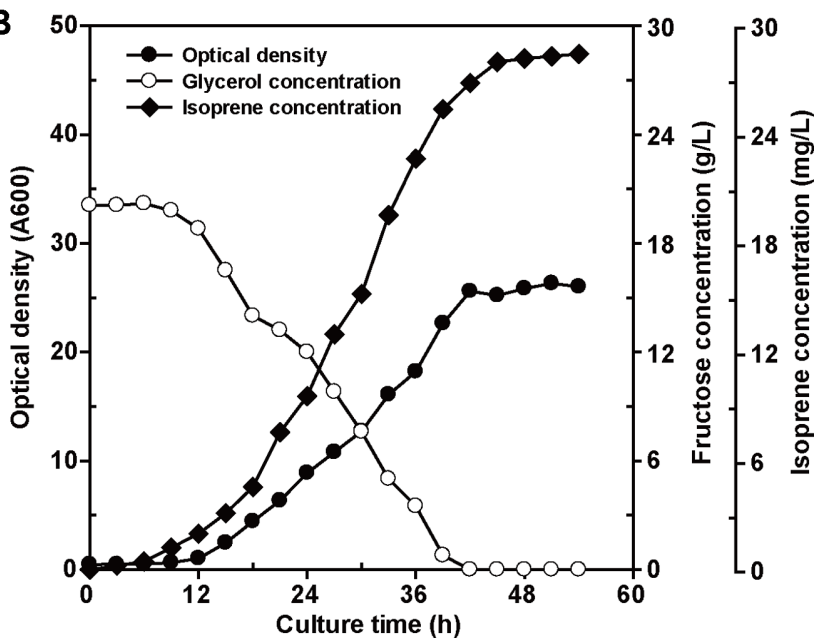

Fig. 3. Time course of 5-L batch cultivation of R. eutropha H16 harboring (A) pRE-MVA3 and (B) pRE-MVA4. phase. The optical density, produced isoprene, specific yield, and carbon yield of $R$. eutropha strains harboring the three-promoter system are summarized in Table 2. As shown in Figs. 3A and 3B, maximal cell growth of R. eutropha RE-MVA7 and RE-MVA8 reached an $\mathrm{OD}_{600}$ of 32.9 and 26.32 at $54 \mathrm{~h}$ of culture, and the isoprene produced was $22.9 \mathrm{mg} / 1$ and $28.45 \mathrm{mg} / \mathrm{l}$, respectively. Isoprene productivity exhibited a rapid decrease upon depletion of the carbon source. Overall, strain RE-MVA8 showed 1.24 times higher isoprene production than RE-MVA7 in 5-L batch culture. In addition, the specific yield and the carbon yield produced relative to the consumed fructose was higher with RE-MVA8 than that obtained with the REMVA8 strain (Table 2, Fig. 3). Based on the results of sealed T-flask cultivation, we could successfully scale-up to a 5 -L batch fermenter for isoprene production using $R$. eutropha containing the three-plasmid system.

\section{Discussion}

Isoprene, a volatile hydrophobic molecule, is one of the major chemical feedstocks in the synthetic chemical industry required for the production of synthetic rubber or plastics and is a future biofuel candidate. The bio-based production of isoprene has a number of advantages; one such advantage is that it can be generated from various kinds of biomass $[13,14]$. Isoprene was mainly produced from renewable carbohydrates such as glucose or glycerol by the engineered E. coli, Bacillus, and S. cerevisiae. However, in recent years, isoprene synthesis studies have been conducted using various strains in order to obtain a production efficiency for commercial use. During the production of isoprene in the photosynthetic apparatus by cyanobacteria, the highest isoprene production yield of $12.3 \mathrm{mg}$ per gram biomass has been reported $(4.2 \mathrm{mg} / 1$ for $96 \mathrm{~h}$ cultivation) [31]. However, biomass or accumulated isoprene could be obtained at a slightly slower rate when using carbon dioxide as a carbon source. Isoprene has been produced by Clostridium ljungdahlii using syngas as an inexpensive carbon source [32]. However, the quantities of produced isoprene and biomass were far lower than that commercially required. $R$. eutropha is commercially employed to produce industrially valuable products in small- or large-scale fermentation processes.

$R$. eutropha is able to convert a significant amount of carbon source into PHB under unfavorable nutrient limitation conditions such as deficiency of nitrogen, phosphate, and oxygen [33]. This ability to produce PHB using excess carbon source may be applied in isoprene production 
because isoprene and PHB share the same production pathway precursor acetyl-CoA. If the conversion pathway of acetyl-CoA toward PHB can be altered to isoprene by genetic modification, $R$. eutropha may be considered an important strain for isoprene bio-production.

Here, we first demonstrated that all the heterologous genes of the mevalonate pathway and isoprene synthase could be utilized for isoprene synthesis by redirecting carbon flow in R. eutropha. The transformants harboring the isoprene production plasmids were evaluated for isoprene production in sealed T-flask and 5-L batch cultures utilizing fructose as the carbon source. In this study, the codon usage of all genes involved in the synthesis was optimized for the efficient production of isoprene through the mevalonate pathway. Using the Lac and J5 promoters, a novel cassette was screened to increase the isoprene production in $R$. eutropha. Codon usage difference for isoprene production between $E$. coli and R. eutropha was observed in sealed T-flask cultures. Strain RE-MVA5 with codon optimization in R. eutropha showed 3.33-fold higher production of isoprene than strain TS-sPt-MVA1 with codon optimization in E. coli using the single Lac promoter system.

Previously, the use of the $\mathrm{P}_{55}$ promoter instead of the $\mathrm{P}_{\mathrm{LAC}}$ promoter increased the protein expression level in R. eutropha [26]. Indeed, the constructed strains RE-MVA5 and RE-MVA6 with $\mathrm{P}_{\mathrm{LAC}}$ and $\mathrm{P}_{\mathrm{J} 5}$ promoters, respectively, produced $1.5 \pm 0.2 \mu \mathrm{g} / 1$ and $1.9 \pm 0.24 \mu \mathrm{g} / 1$ of isoprene, respectively, indicating that increasing the promoter strength enhanced isoprene production. In order to further evaluate the high conversion of carbon flow from acetylCoA to isoprene, the three-promoter system was investigated, and the RE-MVA7 encoding $\mathrm{P}_{\mathrm{LLL}}$ or $\mathrm{P}_{\mathrm{JJJ}}$ were successfully constructed on the basis of the results of recent studies on two-promoter systems to increase protein expression levels or produce PHAs $[27,34]$. Notably, the study on the gene expression efficiency of promoter and ribosome binding sites (RBSs) in $R$. eutropha showed that constitutive promoters with more than 700-fold activity may be located in $R$. eutropha, compared to the native PphaC and RBSs associated with isoprene production by the MEP pathway [35]. In our study, we showed that the maximum titers of $3.8 \pm 0.18 \mu \mathrm{g} / 1$ and $28.45 \mathrm{mg} / 1$ of isoprene were obtained with the $\mathrm{P}_{\text {JJ }}$ system under conditions of sealed T-flask and 5-L batch cultures, respectively, and this promoter system may be applied to produce bio-based products as well as isoprene in engineered R. eutropha.

Although the productivity of isoprene produced in this study is far below that required for commercial production, we have shown that $R$. eutropha may produce potential fuel-based products using various carbon sources such as fatty acids or oils. In particular, the use of non-edible oil such as jatropha is expected to contribute to food or energy reduction issues [36].

To our knowledge, this is the first report on the production of isoprene in $R$. eutropha. To increase isoprene production, we propose enhancing levels of heterologous enzymes, carbon flux optimization, and supply of ATP. In addition, an optimum method of cultivation such as fedbatch, continuous cultivation, and scale-up can be adopted after a thorough cost-benefit analysis in order to make the process technically and economically feasible for industrial application.

\section{Acknowledgments}

We thank Dr. Munjin Kwon for critical reading of this manuscript. This research was supported by grants from the KRIBB (Korea Research Institute of Bioscience \& Biotechnology) Research Initiative Program funded by the government of Korea (KGM-4231612) and from the NextGeneration BioGreen 21 Program (Project No. PJ01368601), Rural Development Administration, Republic of Korea.

\section{Conflict of Interest}

The authors have no financial conflicts of interest to declare.

\section{References}

1. Lee WH, Loo CY, Nomura CT, Sudesh K. 2008. Biosynthesis of polyhydroxyalkanoate copolymers from mixtures of plant oils and 3-hydroxyvalerate precursors. Bioresour. Technol. 99: 6844-6851.

2. Budde CF, Riedel SL, Willis LB, Rha C, Sinskey AJ. 2011. Production of poly(3-hydroxybutyrate-co-3-hydroxyhexanoate) from plant oil by engineered Ralstonia eutropha strains. Appl. Environ. Microbiol. 77: 2847-2854.

3. Doi Y, Kawaguchi Y, Nakamura Y, Kunioka M. 1989. Nuclear magnetic resonance studies of poly(3-Hydroxybutyrate) and polyphosphate metabolism in alcaligenes eutrophus. Appl. Environ. Microbiol. 55: 2932-2938.

4. Tanaka K, Ishizaki A, Kanamaru T, Kawano T. 1995. Production of poly(D-3-hydroxybutyrate) from $\mathrm{CO}(2), \mathrm{H}(2)$, and $\mathrm{O}(2)$ by high cell density autotrophic cultivation of Alcaligenes eutrophus. Biotechnol. Bioeng. 45: 268-275.

5. Park JM, Kim TY, Lee SY. 2011. Genome-scale reconstruction and in silico analysis of the Ralstonia eutropha H16 for 
polyhydroxyalkanoate synthesis, lithoautotrophic growth, and 2-methyl citric acid production. BMC Syst. Biol. 5: 101.

6. Steinbuchel A, Fuchtenbusch B. 1998. Bacterial and other biological systems for polyester production. Trends Biotechnol. 16: 419-427.

7. Schubert P, Steinbuchel A, Schlegel HG. 1988. Cloning of the Alcaligenes eutrophus genes for synthesis of poly-betahydroxybutyric acid (PHB) and synthesis of PHB in Escherichia coli. J. Bacteriol. 170: 5837-5847.

8. Ishizaki A, Tanaka K, Taga N. 2001. Microbial production of poly-D-3-hydroxybutyrate from CO2. Appl. Microbiol. Biotechnol. 57: 6-12.

9. Li H, Opgenorth PH, Wernick DG, Rogers S, Wu TY, Higashide W, et al. 2012. Integrated electromicrobial conversion of $\mathrm{CO} 2$ to higher alcohols. Science 335: 1596.

10. Grousseau E, Lu J, Gorret N, Guillouet SE, Sinskey AJ. 2014. Isopropanol production with engineered Cupriavidus necator as bioproduction platform. Appl. Microbiol. Biotechnol. 98: 4277-4290.

11. Muller J, MacEachran D, Burd H, Sathitsuksanoh N, Bi C, Yeh YC, et al. 2013. Engineering of Ralstonia eutropha H16 for autotrophic and heterotrophic production of methyl ketones. Appl. Environ. Microbiol. 79: 4433-4439.

12. Ward AMS-o-T, GB), Narayanaswamy, Ravichander (Bangalore, IN), Oprins, Arno Johannes Maria (Maastricht, NL), Rajagopalan, Vijayanand (Bangalore, IN), Schaerlaeckens, Egidius Jacoba Maria (Geleen, NL), Velasco Pelaez, Raul (Maastricht, NL). 2016. Process and installation for the conversion of crude oil to petrochemicals having an improved carbon-efficiency. United States patent application 20160369187.

13. Lindberg P, Park S, Melis A. 2010. Engineering a platform for photosynthetic isoprene production in cyanobacteria, using Synechocystis as the model organism. Metab. Eng. 12: 70-79.

14. Bentley FK, Zurbriggen A, Melis A. 2014. Heterologous expression of the mevalonic acid pathway in cyanobacteria enhances endogenous carbon partitioning to isoprene. Mol. Plant. 7: 71-86.

15. Goldstein JL, Brown MS. 1990. Regulation of the mevalonate pathway. Nature 343: 425-430.

16. Rohmer M. 1999. The discovery of a mevalonate-independent pathway for isoprenoid biosynthesis in bacteria, algae and higher plants. Nat. Prod. Rep. 16: 565-574.

17. Lee HW, Park JH, Lee HS, Kim CS, Lee JG, Kim WK, et al. 2019. Development of novel on-line capillary gas chromatography-based analysis method for volatile organic compounds produced by aerobic fermentation. J. Biosci. Bioeng. 127: 121-127.

18. Xue J, Ahring BK. 2011. Enhancing isoprene production by genetic modification of the 1-deoxy-d-xylulose-5-phosphate pathway in Bacillus subtilis. Appl. Environ. Microbiol. 77: 2399-2405.
19. Lv X, Xie W, Lu W, Guo F, Gu J, Yu H, et al. 2014. Enhanced isoprene biosynthesis in Saccharomyces cerevisiae by engineering of the native acetyl-CoA and mevalonic acid pathways with a push-pull-restrain strategy. J. Biotechnol. 186: 128-136.

20. Eroglu E, Melis A. 2010. Extracellular terpenoid hydrocarbon extraction and quantitation from the green microalgae Botryococcus braunii var. Showa. Bioresour. Technol. 101: 23592366.

21. Seemann M, Campos N, Rodriguez-Concepción M, Ibañez E, Duvold T, Tritsch D, et al. 2002. Isoprenoid biosynthesis in Escherichia coli via the methylerythritol phosphate pathway: enzymatic conversion of methylerythritol cyclodiphosphate into a phosphorylated derivative of (E)-2-methylbut-2-ene1,4-diol. Tetrahedron. Lett. 43: 1413-1415.

22. Farmer WR, Liao JC. 2001. Precursor balancing for metabolic engineering of lycopene production in Escherichia coli. Biotechnol. Prog. 17: 57-61.

23. Kajiwara S, Fraser PD, Kondo K, Misawa N. 1997. Expression of an exogenous isopentenyl diphosphate isomerase gene enhances isoprenoid biosynthesis in Escherichia coli. Biochem. J. 324(Pt 2): 421-426.

24. Martin VJ, Pitera DJ, Withers ST, Newman JD, Keasling JD. 2003. Engineering a mevalonate pathway in Escherichia coli for production of terpenoids. Nat. Biotechnol. 21: 796-802.

25. Zurbriggen A, Kirst H, Melis A. 2012. Isoprene production via the mevalonic acid pathway in Escherichia coli (Bacteria). BioEnergy Res. 5: 814-828.

26. Gruber S, Hagen J, Schwab H, Koefinger P. 2014. Versatile and stable vectors for efficient gene expression in Ralstonia eutropha H16. J. Biotechnol. 186: 74-82.

27. Kim KJ, Kim HE, Lee KH, Han W, Yi MJ, Jeong J, et al. 2004. Two-promoter vector is highly efficient for overproduction of protein complexes. Protein Sci. 13: 1698-1703.

28. Kovach ME, Elzer PH, Hill DS, Robertson GT, Farris MA, Roop RM, 2nd, et al. 1995. Four new derivatives of the broad-host-range cloning vector pBBR1MCS, carrying different antibiotic-resistance cassettes. Gene 166: 175-176.

29. Lee HW, Lee HS, Kim CS, Lee JG, Kim WK, Lee EG, et al. 2018. Enhancement of L-threonine production by controlling sequential carbon-nitrogen ratios during fermentation. J. Microbiol. Biotechnol. 28: 293-297.

30. Wu H-S, Tsai M-J. 2004. Kinetics of tributyrin hydrolysis by lipase. Enzyme Microbial Technol. 35: 488-493.

31. Chaves JE, Melis A. 2018. Biotechnology of cyanobacterial isoprene production. Appl. Microbiol. Biotechnol. 102: 64516458.

32. Diner BA, Fan J, Scotcher MC, Wells DH, Whited GM. 2018. Synthesis of heterologous mevalonic acid pathway enzymes in clostridium ljungdahlii for the conversion of fructose and of syngas to mevalonate and isoprene. Appl. Environ. Microbiol. 84. pii: e01723-17. 
33. Koller M, Atlić A, Dias M, Reiterer A, Braunegg G. 2010. Microbial PHA Production from Waste Raw Materials, pp. 85-119. In Chen GG-Q (ed.), Plastics from Bacteria: Natural Functions and Applications, Ed. Springer Berlin Heidelberg, Berlin, Heidelberg

34. Fukui T, Ohsawa K, Mifune J, Orita I, Nakamura S. 2011. Evaluation of promoters for gene expression in polyhydroxyalkanoate-producing Cupriavidus necator H16. Appl. Microbiol. Biotechnol. 89: 1527-1536.
35. Alagesan S, Hanko EKR, Malys N, Ehsaan M, Winzer K, Minton NP. 2018. Functional genetic elements for controlling gene expression in Cupriavidus necator H16. Appl. Environ. Microbiol. 84. pii: e00878-18.

36. Batcha AF, Prasad DM, Khan MR, Abdullah H. 2014. Biosynthesis of poly(3-hydroxybutyrate) (PHB) by Cupriavidus necator H16 from jatropha oil as carbon source. Bioprocess Biosyst. Eng. 37: 943-951. 